\title{
Yeast Artificial Chromosome
}

National Cancer Institute

\section{Source}

National Cancer Institute. Yeast Artificial Chromosome. NCI Thesaurus. Code C1457.

A cloning vector based upon elements of yeast chromosomes. At a minimum these include: yeast telomeres, a yeast centromere, and a yeast autonomously replicating sequence (ARS). Such vectors are propagated in yeast and then transferred into a target eukaryotic cell where the yeast sequences can be recognized and will maintain the molecule through cell division. These vectors can maintain extremely large amounts of foreign DNA. YACs are maintained as linear molecules 